\title{
Aplicação das técnicas aproximativas de diferenças finitas e elementos finitos em problema de natureza parabólica não linear
}

\author{
Neves, N. S. ${ }^{1 *}$, Pinheiro, V. P. ${ }^{2}$, Almeida, L. M. ${ }^{3}$ \\ ${ }^{1}$ COPPE - Programa de Engenharia Civil, Universidade Federal do Rio de Janeiro, RJ, Brasil. \\ ${ }^{2}$ PPGEM - Programa de Pós-graduação em Engenharia Mecânica, UFES, ES, Brasil. \\ ${ }^{3}$ Programa de Iniciação Científica, PRPPGE, Universidade Vila Velha, ES, Brasil \\ *natansian@ufrj.br
}

\begin{abstract}
Resumo
O presente artigo busca realizar uma análise térmica bidimensional em um domínio regular representativo de um elemento estrutural de concreto exposto a um fluxo de calor não linear. Sendo assim, aplica-se técnicas numéricas para estimar o campo potencial do fenômeno de interesse descrito pela equação de difusão de calor não linear transiente. As análises e estratégias empregadas para as soluções são direcionadas pela utilização do método de elementos finitos e do método das diferenças finitas. As implementações computacionais são realizadas em ambiente Matlab. Em síntese, os métodos numéricos utilizados na solução do problema apresentam resultados satisfatórios em comparação com os dados disponiveis na literatura, representando adequadamente o comportamento física do fenômeno estudado.
\end{abstract}

Palavras-chave: MDF, MEF, Equação parabólica não linear, Programa computacional.

\section{Introdução}

Os conceitos de transferência de calor possuem importantes aplicações em diversos campos da engenharia, interligados aos problemas relacionados a área da termodinâmica, mecânicas de fluidos e das estruturas sob condição de incêndio, onde se exige a construção de modelos matemáticos e numéricos com inúmeros complexidades físicas.

Os elementos estruturais quando expostos a elevados gradientes térmicos apresentam severas reduções nas propriedades físicas e mecânica, acarretando em possíveis prejuízos a vida e ao patrimônio. Desta maneira, para minimizar os riscos nessas condições, um dos importantes fatores para projeto é o conhecimento do campo térmico na seção transversal das estruturas em situação de incêndio [1].

\section{Teoria Geral e Aplicação}

Para aplicar os métodos numéricos de natureza diferencial ou integral, deve-se conhecer, previamente, o modelo teórico que governa o fenômeno físico [2]. No caso da condução de calor não linear em regime transiente, a equação diferencial do problema é dado a seguir.

$$
k(u)\left[\frac{\partial^{2} u}{\partial x^{2}}+\frac{\partial^{2} u}{\partial y^{2}}\right]=c \rho(u) \frac{\partial u}{\partial t}
$$

Onde $u$ é o campo potencial de temperatura, $\rho$ é a massa específica, $c$ é o calor específico e $k$ é a condutividade térmica do material, considerando um meio isotrópico. 

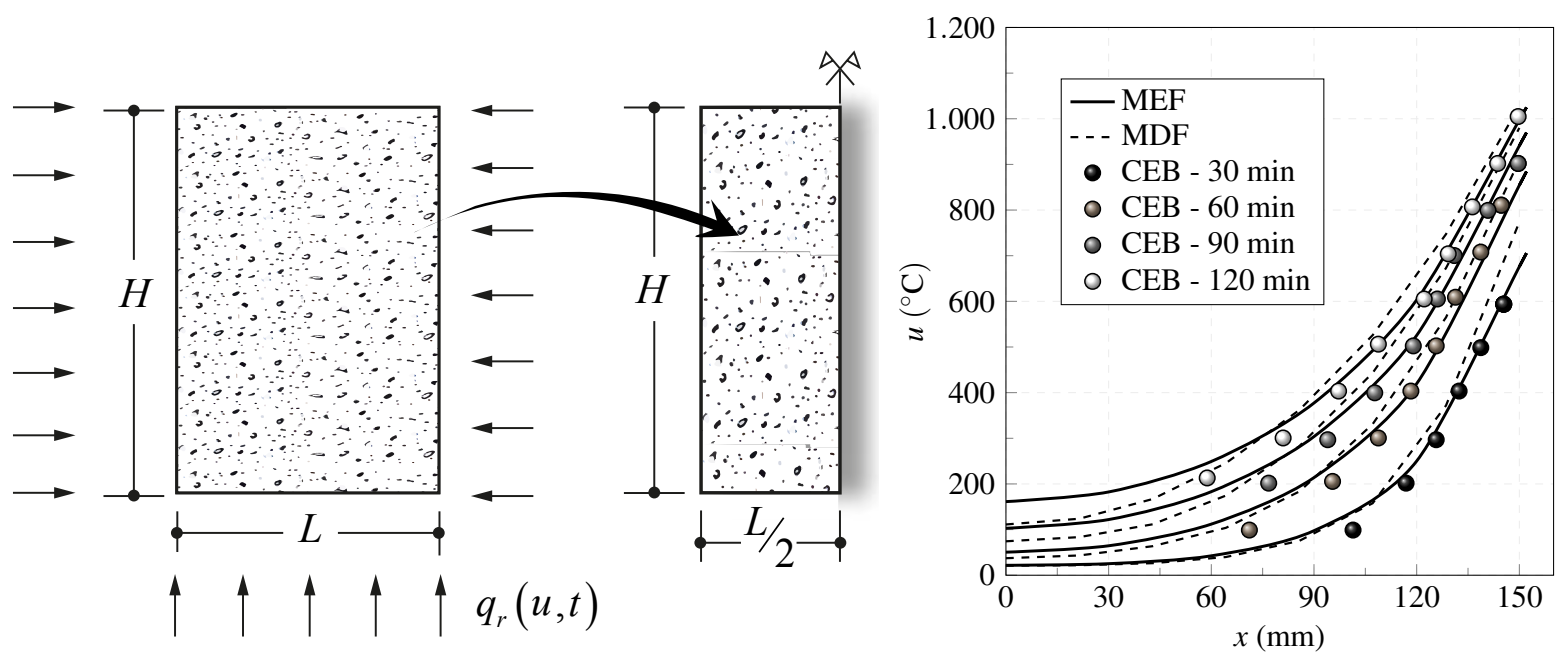

Figura 1: Características gerais (esquerda) e resultados numéricos do problema (direita).

As funções que descrevem o comportamento das propriedades com aumento de temperatura são descritos no EN 1992 [3]. A condição inicial do problema é tomada igual $u(x, y, 0)=u_{0}$. Além disso, aplica-se nas fronteiras do domínio um fluxo de calor combinado de convecção-radiação, conforme posto na Equação (2).

$$
q_{r}=\lambda_{c}\left(u-u_{\infty}\right)+\varepsilon \sigma\left(u^{4}-u_{\infty}{ }^{4}\right)
$$

Em que $\lambda_{c}$ é o coeficiente de convecção, $\varepsilon$ é a emissividade, $\sigma$ conhecido como constate de Stefan-Boltzmann e ainda, $u_{\infty}$ é o valor de temperatura do fluído, descrito conforme a curva ISO 834 [4]. Desta forma, a solução numérica com base no método das diferenças finitas (MDF), segue uma lógica simples e direta, uma vez que a técnica aproxima os operadores diferenciais por expressões algébrica entre os pontos adjacentes da malha [5]. Desta forma, aplicando as aproximações centrais e progressivas para os operadores diferenciais espaçais e o operador temporal, respetivamente, chega-se na seguinte expressão:

$$
\begin{array}{r}
u_{i+1, j}^{n+1}+u_{i-1, j}^{n+1}+\left(-4-\frac{h^{2} \rho c}{k \Delta t}\right) u_{i, j}^{n+1}+ \\
\cdots u_{i, j+1}^{n+1}+u_{i, j-1}^{n+1}=-\left(\frac{h^{2} \rho c}{k \Delta t}\right) u_{i, j}^{n}
\end{array}
$$

A solução via elementos finitos (MEF) é fundamentado no método de resíduos pon- derados de Galerkin. Aplicando os teoremas do cálculo diferencial-integral e com base na aproximação do campo primal por uma combinação linear entre coeficientes e funções de forma [6], chega-se na forma fraca discreta do problema.

$$
\begin{gathered}
\int_{\Gamma} q_{\mathbf{n}} N_{i} d \Gamma=\sum_{j=1}^{n}\left[\rho c \int_{\Omega} N_{j} N_{i} d \Omega\right] \dot{u}_{j}+ \\
\sum_{j=1}^{n}\left[k \int_{\Omega}\left(\frac{\partial N_{j}}{\partial x} \frac{d N_{i}}{d x}+\frac{\partial N_{j}}{\partial y} \frac{d N_{i}}{d y}\right) d \Omega\right] u_{j}
\end{gathered}
$$

As funções de forma $N$ variam de acordo com escolha do tipo de elemento finito utilizado na formulação, considerando os elementos triangulares lineares, no sistema local de referência, usa-se as seguintes funções:

$$
\begin{array}{r}
N_{1}(\xi, \eta)=\xi \\
N_{2}(\xi, \eta)=\eta \\
N_{3}(\xi, \eta)=1-\xi-\eta
\end{array}
$$

Além disso, o operador derivada temporal é aproximada com auxílio da técnica de diferenças finitas, conforme pode ser visto em detalhes no trabalho de Neves [7].

$$
[\mathbf{K}]\{\mathbf{u}\}=\{\mathbf{F}\}
$$

Independente da técnica utilizada para solução da equação de difusão, chega-se em um sistema algébrico convencional, conforme 


\section{Blucher Proceedings XI Encontro Científico de Física Aplicada}
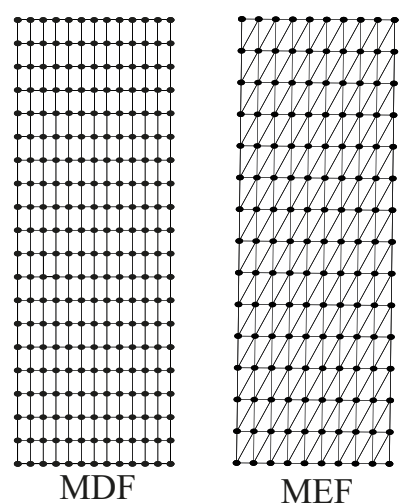

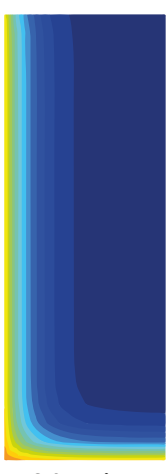

$30 \mathrm{~min}$

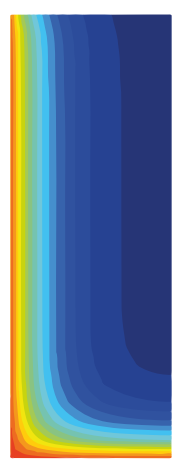

$60 \mathrm{~min}$

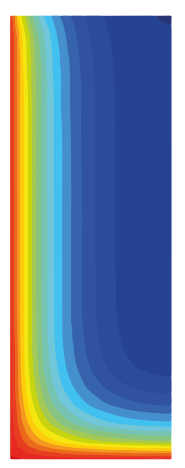

$90 \mathrm{~min}$

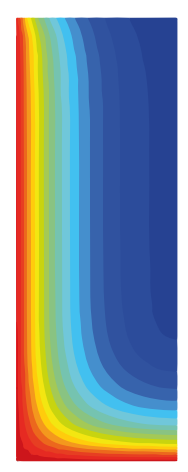

$120 \mathrm{~min}$

Figura 2: Malha numérica de MDF e MEF, e campo térmica da seção transversal.

mostra a Equação (8). Vale ressaltar que por causa da natureza não linear do modelo, a solução parte de um processo incrementaliterativo.

O caso teste é composto de uma viga de concreto de $600 \times 300 \mathrm{~mm}$ submetida ao fluxo combinado de convecção-radiação em três faces do domínio, onde utiliza-se a simetria do problema, conforme representado na Figura 1. Na Figura 2, mostra-se a malha numérica de elementos finitos e diferentes finitas utilizadas nas simulações, bem como a distribuição de temperatura na seção transversal.

Os resultados são medidos ao longo da linha média da estrutura para 30,60, 90 e 120 min de exposição ao fogo, sendo que os valores obtidos pelas técnicas numéricas são calibrados com os dados obtidos na CEB [8]. Observa-se que as curvas dos perfis de temperatura ao longo da largura da viga apresentam valores abaixo de $200^{\circ} \mathrm{C}$, para regiões próximas ao centro do viga, enquanto as bordas expostas ao fogo podem atingir níveis de $1000^{\circ} \mathrm{C}$, indicando valores favoráveis a segurança no interior da viga de concreto.

\section{Conclusão}

O presente artigo visou investigar o comportamento térmico de uma viga retangular de concreto sob ação de altos gradientes térmicos com base nos procedimentos de elementos finitos e de diferenças finitas. Ao fim, foi possível concluir que os resultados apresentados, por ambas os métodos, foram satisfatório e bem ajustados com a solução normativa de referência. Além disso, pode-se afirmar que a formulação do MEF apresenta uma generalizada de aplicação, enquanto a formulação clássica do MDF fica restrito as condições e características regulares do domínio.

\section{Referências}

[1] PURKISS, J. A.; LI, L. Y. Fire safety engineering design of structures. CRC press, 2013.

[2] MEEK, J. L. Computer methods in structural analysis. CRC Press, 2017.

[3] EN 1992-1-2. Design of concrete structures: General rules - Structural fire design, 2004.

[4] ISO 834: Fire resistance tests-elements of building construction. Geneva, 1999 .

[5] ÖZISIK, M. N. et al. Finite difference methods in heat transfer. New York: CRC press, 2017.

[6] REDDY, J. N. An introduction to the finite element method. New York, 1993.

[7] NEVES, N. S. Modelo computacional avançado para análise de estruturas sob ação de gradientes térmicos. UFES, 2019.

[8] CEB. Design of concrete structures for fire resistence. Paris, 1982. 